\title{
Contemporary Understanding of miRNA-Based Regulation of Secondary Metabolites Biosynthesis in Plants
}

\author{
Om P. Gupta ${ }^{1 * t}$, Suhas G. Karkute ${ }^{2}$, Sagar Banerjee ${ }^{3}$, Nand L. Meena ${ }^{4}$ and Anil Dahuja ${ }^{3}$ \\ ${ }^{1}$ Division of Quality and Basic Sciences, ICAR-Indian Institute of Wheat and Barley Research, Karnal, India, ${ }^{2}$ Division of \\ Vegetable Improvement, ICAR-Indian Institute of Vegetable Research, Varanasi, India, ${ }^{3}$ Division of Biochemistry, ICAR-Indian \\ Agricultural Research Institute, New Delhi, India, ${ }^{4}$ Division of Basic Sciences, ICAR-Indian Institute of Millets Research, \\ Hyderabad, India
}

Plant's secondary metabolites such as flavonoids, terpenoids, and alkaloids etc. are known for their role in the defense against various insects-pests of plants and for medicinal benefits in human. Due to the immense biological importance of these phytochemicals, understanding the regulation of their biosynthetic pathway is crucial. In the recent past, advancement in the molecular technologies has enabled us to better understand the proteins, enzymes, genes, etc. involved in the biosynthetic pathway of the secondary metabolites. miRNAs are magical, tiny, non-coding ribonucleotides that function as critical regulators of gene expression in eukaryotes. Despite the accumulated knowledge of the miRNA-mediated regulation of several processes, the involvement of miRNAs in regulating secondary plant product biosynthesis is still poorly understood. Here, we summarize the recent progress made in the area of identification and characterizations of miRNAs involved in regulating the biosynthesis of secondary metabolites in plants and discuss the future perspectives for designing the viable strategies for their targeted manipulation.

Keywords: miRNAs, terpenoids, alkaloids, flavonoids, phenolics, glycosides

\section{INTRODUCTION}

Since the age of human civilization, plants are used as a source of nutrition and medicine, which is evidenced by the numerous texts from China and India (Kirtikar and Basu, 1918; Tang and Eisenbrand, 1992). The nutritional and medicinal properties of the plants are due to the presence of numerous metabolites. These metabolites are of two types: primary and secondary. Unlike primary metabolites, secondary metabolites are a huge group of phytochemicals, which are not directly involved in plant's vital processes such as growth, development, and reproduction (Fraenkel, 1959) but they are major components in defense mechanism of plants in order to protect them from any possible harm in the ecological environment (Stamp, 2003) and other interspecies protection (Samuni-Blank et al., 2012). Humans have exploited secondary metabolites in the form of flavoring agents, fragrances, insecticides, dyes, drugs, etc., More than 100,000 phytochemicals have been isolated from different plant sources so far (Mahajan et al., 2011). These secondary metabolites are broadly categorized as terpenoids, alkaloids, phenolics, glycosides, tannins, and saponins (Verpoorte, 1998). These phytochemicals are synthesized in the plants for a specialized need in a specific set of ecological conditions as their biosynthesis are highly energy consuming. This kind of biosynthesis and accumulation behavior of secondary metabolites in plants is the 
result of tight regulation of their biosynthetic machinery. Metabolic engineering may further pave a way for enhancing biosynthesis of economically important phytochemicals or for producing desired combinations of such chemicals. One of the ways to tinker with biosynthetic pathways is through modulating miRNA levels as miRNAs are the ultimate regulators in plants.

miRNAs are small (21-24 nucleotides), non-coding, riboregulators that regulate gene expression in eukaryotes (Jones-Rhoades et al., 2006). miRNA is transcribed by RNA polymerase II as a precursor RNA known as the primary miRNA (pri-miRNA), which is subsequently processed by DICER-LIKE 1 (DCL1) to release the mature miRNAs. These mature miRNAs are then loaded into the RISC complex to bind mRNAs for cleavage (Jones-Rhoades et al., 2006). miRNAs are well-known molecules for their role in regulating various plants processes under biotic and abiotic stresses (Gupta et al., 2014a,b; Shriram et al., 2016). Recently, various reports suggested their roles in regulating the biosynthesis and accumulation of secondary metabolites in plants (see review Bulgakov and Avramenko, 2015). In the present review, we have updated the knowledge about present understanding on miRNAs based regulation of biosynthesis and accumulation of secondary metabolites in plants.

\section{ROLE OF miRNAs IN FLAVONOID BIOSYNTHESIS}

Flavonoids such as flavonols, flavones, isoflavones, anthocyanins, proanthocyanidins, and phlobaphene pigments are low molecular weight phenylpropanoid compounds which are widely distributed throughout the plant kingdom (Taylor and Grotewold, 2005; Lepiniec et al., 2006; Buer et al., 2010). These polyphenolic metabolites play a variety of significant biological roles such as protection against UV radiation, as signaling molecules, as phytoalexins in plant-microbe interaction, and as regulators of phytohormones such as auxin transport in plants (Santelia et al., 2008; Buer et al., 2010). The flavonoid backbone is synthesized by the central phenylpropanoid pathway and different flavonoid metabolites share common enzymes and substrates. Phenylpropanoid pathway is one of the most extensively studied pathways of secondary metabolites for transcriptional regulation in plants (Quattrocchio et al., 2006; Stracke et al., 2007; Li, 2014). In the past few years, scientific endeavors are directed toward understanding the posttranscriptional regulation of this pathway involving miRNAs. The schematic representation of the general phenylpropanoid pathway leading to major branches of flavonoid biosynthesis and their possible interaction with miRNAs has been depicted in Figure 1A.

About 17 SQUAMOSA PROMOTER BINDING PROTEINLIKE (SPL) proteins are encoded by the Arabidopsis genome (Riese et al., 2007). These SPL transcription factors are reported to affect numerous processes of plant growth and development, such as vegetative phase transition by enhancing the expression of miRNA172, flowering induction by LEAFY and MADS box genes, embryonic development, cell size, trichome formation, and fertility (Wu et al., 2009; Yamaguchi et al., 2009; Xing et al., 2010; Yu et al., 2010). In addition, miR156 targeted SPL9 protein has been shown to regulate the metabolic flux during flavonoid biosynthetic pathway. Anthocyanins accumulate in an acropetal manner in Arabidopsis stems, with the highest level at the junction between the stem and the rosette leaves. This array of anthocyanin accumulation is regulated by the miR156 targeted SPL9 gene in Arabidopsis (Gou et al., 2011). The tissues having high anthocyanin concentration accumulate higher levels of miRNA156 leading to reduced SPL activity which in turn enhance the expression of $\mathrm{F}^{\prime} \mathrm{H}$, DFR, and other anthocyanin biosynthetic genes. As a result, dihydroflavonols are directed into the anthocyanin branch. On the other hand, expression of SPLs gradually increases along the growing stem because miR156 levels decline as the plant progresses during development (Gou et al., 2011). Therefore, increased accumulation of SPL leads to decreased expression of anthocyanin biosynthetic genes resulting in the increased production of flavonols by FLS. It has been demonstrated that MYB-bHLH-WD40 transcriptional activation complex is destabilized by SPL9, a target of miRNA156, by competing with bHLHs for their binding to PAP1 which in turn inhibits expression of anthocyanin biosynthetic genes (anthocyanidin synthase, flavanone 3hydroxylase, dihydroflavonol reductase, and UDP-glucosyl transferase $75 \mathrm{C} 1$ etc.) influencing anthocyanin accumulation in Arabidopsis (Gou et al., 2011). Similarly, miRNA156SPL9 pair influences anthocyanin production by targeting dihydroflavonol 4-reductase (Cui et al., 2014). Therefore, an antagonistic relationship exists between anthocyanin and flavonol biosynthesis in Arabidopsis. Recently, Biswas et al. (2016) have computationally identified several miRNAs such as miR172i, miR829.1, miR1438, miR1873, and miR5532 targeting mRNAs coding for enzymes of phenylpropanoid pathway, such as 4-coumarate-CoA ligase, Chalcone synthase, CaffeoylCoA O-methyl transferase, Dihydroflavonol 4-reductase C, 2-hydroxyisoflavanone dehydratase respectively in Podophyllum hexandrum (Table 1). Overexpression of miR8154 and miR5298b in sub-cultured Taxus cell lines revealed their crucial role in the regulation of taxol, phenylpropanoid, and flavonoid biosynthesis pathways (Zhang et al., 2015). Similarly, several other miRNAs of phenylpropanoid pathway, such as miR395p-3p/ targeting bHLH mRNA in D. kaki (Luo et al., 2015), miR396b and miR828a targeting mRNAs coding for Kaempferol 3-O-beta$\mathrm{D}$-galactosyltransferase and anthocyanin regulatory $\mathrm{C} 1$ protein respectively in $R$. serpentina (Prakash et al., 2016), miR858a targeting R2R3-MYB mRNA in A. thaliana (Sharma et al., 2016), miR6194 targeting Flavanone 3b-hydroxylase mRNA (F3H) in H. caspica (Yang et al., 2015), miR1061-3p and miR1318 in pear fruit (Wu et al., 2014) etc., (Table 1) have been reported.

Further, the use of advanced computational tools complementing the experimental methods has accelerated the accumulation of reports on new as well as existing miRNAs implying their regulatory role during flavonoid pathway in plants. Therefore, further work on functional characterization of these tiny miRNAs-target networks using reverse genetic approach would certainly pave a way for understanding posttranscriptional regulatory mechanism of the flavonoid pathway. 


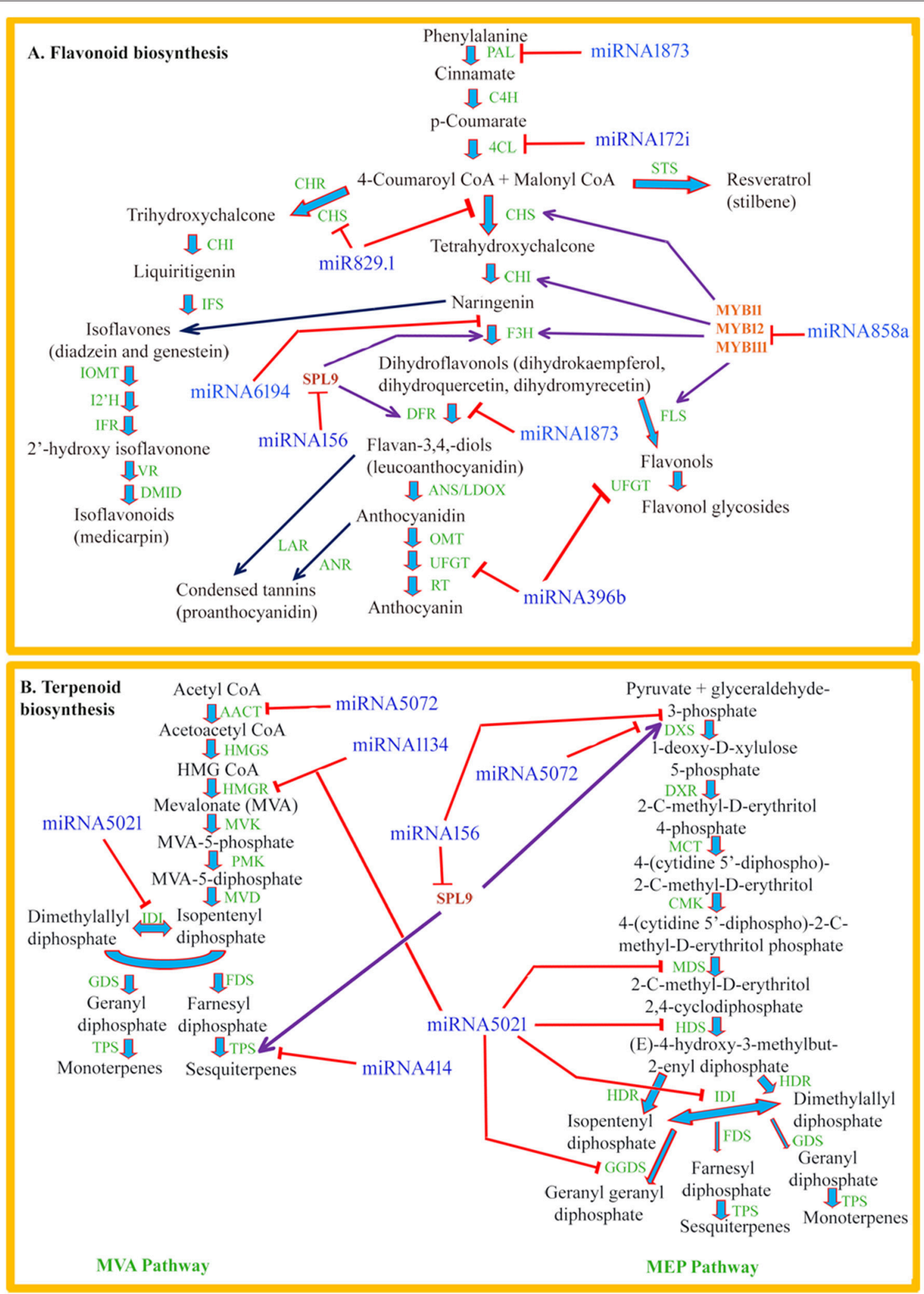

FIGURE 1 | (A) Schematic representation of the general phenylpropanoid pathway leading to major branches of flavonoid biosynthesis and their possible interaction with miRNAs. Phe ammonia-lyase (PAL); cinnamate-4-hydroxylase (C4H); 4-coumaroyl:CoA-ligase (4CL); chalcone reductase (CHR), chalcone synthase (CHS); 


\section{FIGURE 1 | Continued}

stilbene synthase (STS); chalcone isomerase (CHI); flavanone 3-hydroxylase (F3H); isoflavone synthase (IFS); dihydroflavonol 4-reductase (DFR); isoflavone O-methyltransferase (IOMT); isoflavone $2^{\prime}$-hydroxylase (I2' H); isoflavone reductase (IFR); vestitone reductase (VR); $2^{\prime}$-dihydroxy, 49-methoxyisoflavanol dehydratase (DMID); leucoanthocyanidin dioxygenase (LDOX); O-methyltransferase (OMT); UDPG-flavonoid glucosyl transferase (UFGT); rhamnosyl transferase (RT); flavonol synthase (FLS); leucoanthocyanidin reductase (LAR); anthocyanidin reductase (ANR); anthocyanidin synthase (ANS). (B) Schematic representation of biosynthetic pathway of volatile terpenoid and their possible interaction with miRNAs. acetoacetyl-CoA thiolase (AACT); HMG-CoA synthase (HMGS); HMG-CoA reductase (HMGR); mevalonate kinase (MVK); phosphomevalonate kinase (PMK); mevalonate diphosphate decarboxylase (MVD); isopentenyl diphosphate isomerase (IDI); geranyl diphosphate synthase (GDS); farnesyl diphosphate synthase (FDS); terpene synthase (TPS); DOXP synthase (DXS); DOXP reductoisomerase (DXR); 2-C-methyl-D-erythritol 4-phosphate cytidylyltransferase (MCT); CDP-ME kinase (CMK); 2-C-methyl-D-erythritol 2,4-cyclodiphosphate synthase (MDS); (E)-4-hydroxy-3-methylbut-2-enyl diphosphate synthase (HDS); (E)-4-hydroxy-3-methylbut-2-enyl diphosphate reductase (HDR); geranyl geranyl diphosphate synthase (GGDS).

This information could further be used for metabolic engineering of the entire pathway for human benefits.

\section{ROLE OF MIRNAS IN TERPENOID BIOSYNTHESIS}

Owing to their numerous biological roles, isoprene (C5), monoterpenes (C10), and sesquiterpenes (C15) establish the biggest class of plant volatile compounds. In plants, these volatile compounds act as defense molecules against biotic stresses, attracts pollinators and seed disseminators, and help improve thermo-tolerance (Dudareva et al., 2006). In addition, they are used as aroma compounds and natural flavor enhancers which have the beneficial impact on human health (Wagner and Elmadfa, 2003). Considering the importance of these compounds, understanding the regulatory schema of their biosynthetic pathway and accumulation stands on priority. These volatile compounds are synthesized from isopentenyl diphosphate (IPP) and dimethylallyl diphosphate (DMAPP), which are derived from two alternate biosynthetic pathways localized in different subcellular compartments. During the past several years, there has been a significant progress in identification and characterization of genes and enzymes involved in the biosynthesis of volatile terpenoids (Figure 1B), determination of their spatiotemporal expression and compartmentalization, and metabolic engineering. However, the regulatory role of miRNAs in their biosynthesis and accumulation is poorly understood, which opens a new window for further investigations.

Terpene synthases (TPSs) Catalyses the conversion of farnesyl diphosphate (FPP) into sesquiterpenes (C15). Transcription factor SPL9, the target of miRNA156, directly binds to and activates promoter of terpene synthases 21 (TPS21) gene and positively regulates its transcription thereby regulating the synthesis of sesquiterpenoid (Yu et al., 2015). Similarly, miR4995 was predicted to target mRNA of an enzyme 3-deoxy-7phosphoheptulonate synthase, which is involved in the picroside biosynthetic pathway in a medicinal herb P. kurroa (Vashisht et al., 2015). In addition, Saifi et al. (2015), have mined and validated 11 miRNAs which are involved in steviol glycoside biosynthetic pathway (Table 1) in Stevia and established the relationship pattern with the expression levels of their target mRNAs as well as steviol glycoside contents. Using NGS technology, several miRNAs involved in the sesquiterpene biosynthesis pathway have been mapped and validated in $X$. strumarium. For example, mRNAs of the upstream enzymes in the pathways of terpenoid biosynthesis, including 1deoxy-D-xylulose 5-phosphate synthase (DXS), 3-hydroxy-3methylglutaryl coenzyme A reductase (HMGR), isopentenyl diphosphate (IPP)/dimethylallyl diphosphate (DMAPP) synthase (IDS), and isopenteyl diphosphate isomerase (IDI) were predicted to be targeted by miR7539, miR5021, and miR1134 (Fan et al., 2015). The complete list of miRNAs and their target genes have been provided in Table 1. Most recently, bioinformatics approaches have been utilized to mine miRNAs involved in terpenoid metabolism in Mentha spp. (Singh et al., 2016a), Ginger (Singh et al., 2016b), C. roseus (Pani and Mahapatra, 2013), and P. hexandrum (Biswas et al., 2016; Table 1).

\section{THE ROLE OF miRNAs IN THE REGULATING BIOSYNTHESIS OF ALKALOID AND OTHER N-CONTAINING METABOLITES}

Alkaloids are nitrogen containing low molecular-weight compounds which are mostly derived from amino acids. They are known to play significant roles in defense against herbivores and pathogens and are being widely used as pharmaceuticals, stimulants, narcotics, and poisons. Unlike other secondary metabolites, this class is highly diverse and heterogenous in nature and around $\sim 12,000$ alkaloids have been characterized till date (Ziegler and Facchini, 2008). These compounds are synthesized through diverse metabolic pathways. Recent genome based technological advancement have led us to add to on our current understanding of their biosynthetic pathways and regulation. However, knowledge on the role of miRNAs during alkaloid biosynthesis and accumulation in plant kingdom has just started to proliferate.

Boke and his coworkers in 2014 have extensively worked on regulation of the alkaloid biosynthesis by miRNA in opium poppy. They identified pso-miR13, pso-miR2161, and pso-miR408 as potential miRNAs involved in the alkaloid biosynthetic pathway. Pso-miRNA2161 targets the mRNA of gene encoding S-adenosyl-L-methionine: 30-hydroxy$\mathrm{N}$-methylcoclaurine 40-O-methyltransferase 2 (4O $\mathrm{MT}$ ) enzyme which converts S-norcoclaurine into S-reticuline, an intermediate molecule in benzylisoquinoline alkaloids (BIA) biosynthesis. Similarly, pso-miR13 targets mRNA of 7-Omethyltransferase (7O MT) gene, which converts S-reticuline to morphinan alkaloids. pso-miR408 targets mRNA of reticuline 

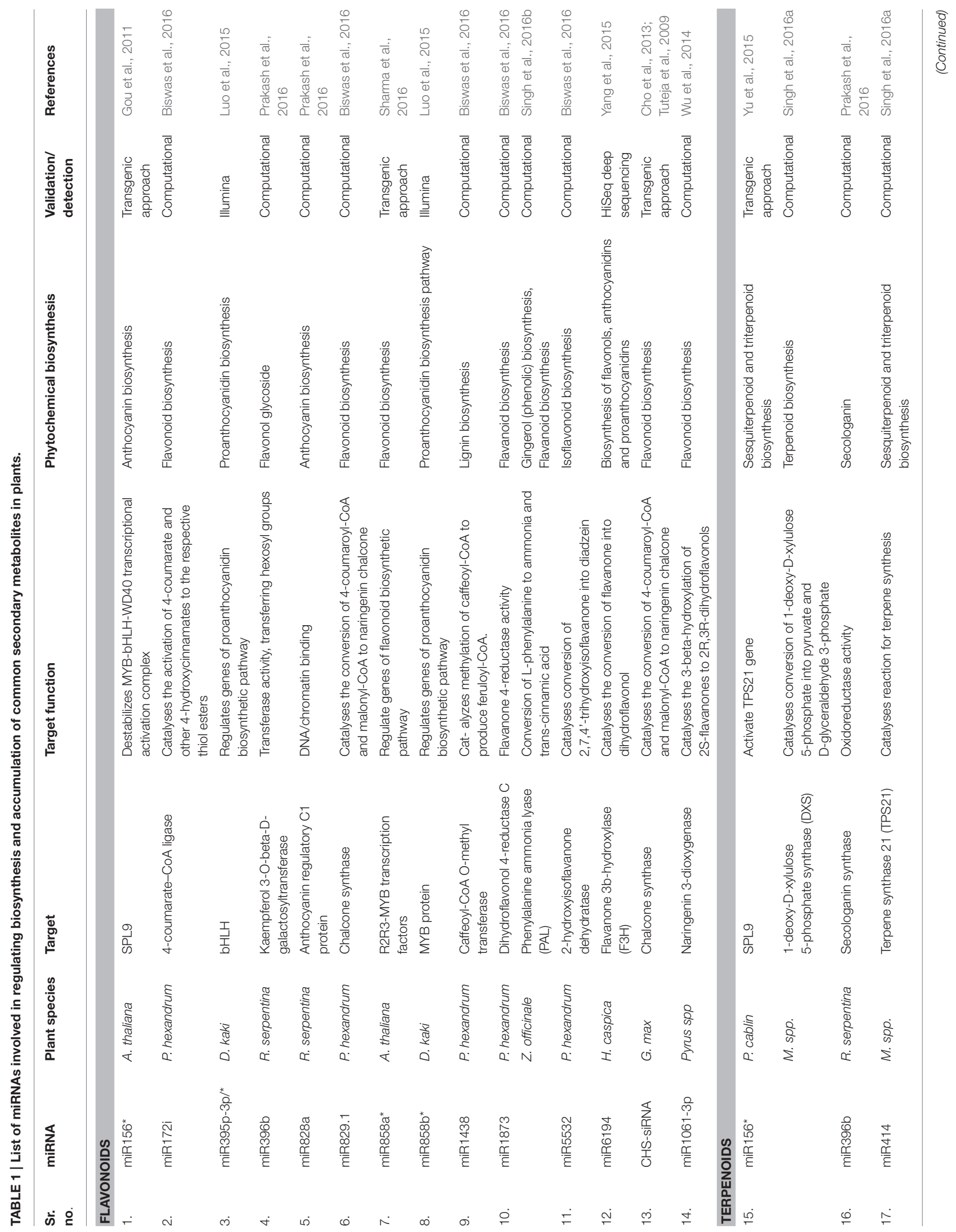


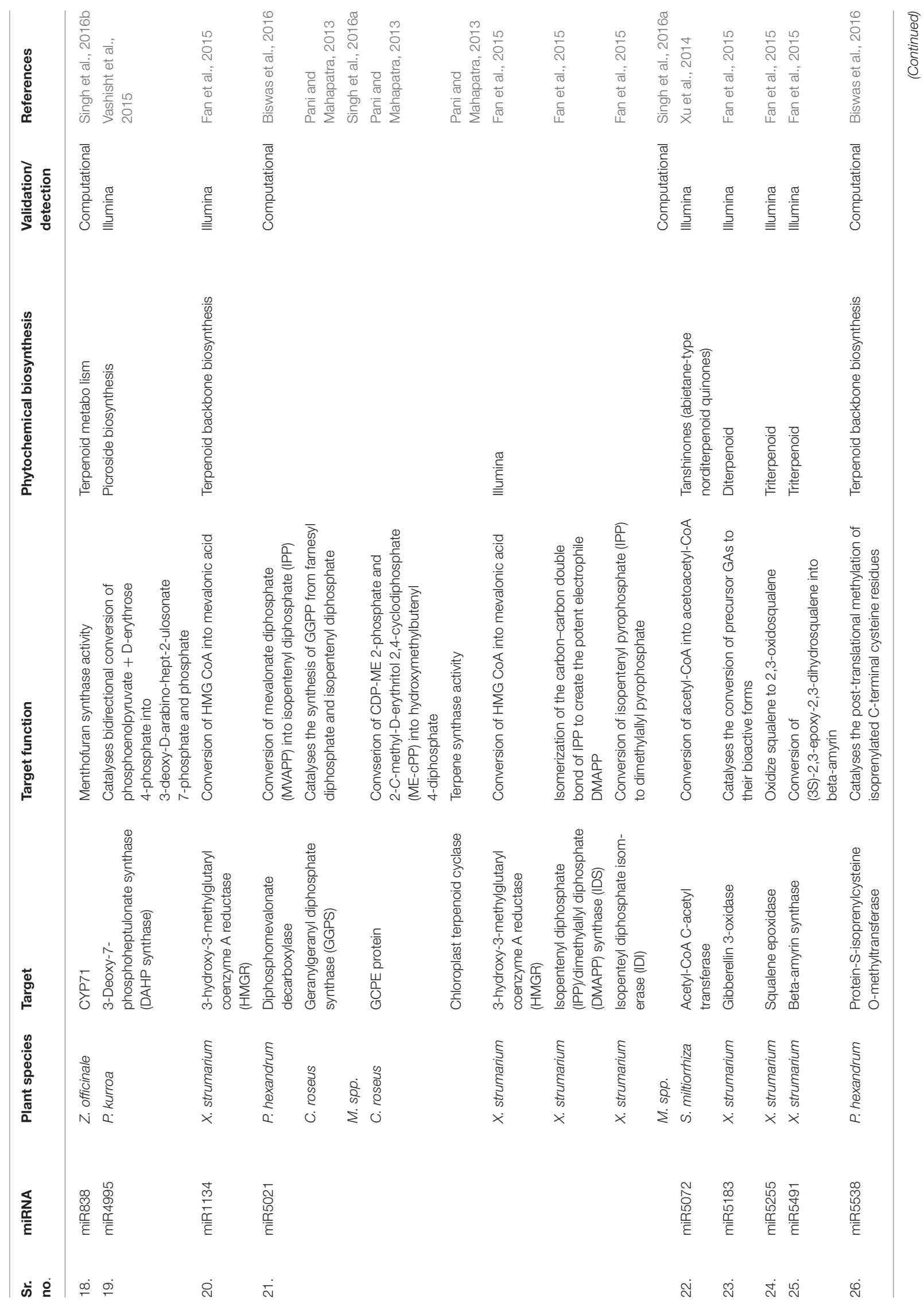



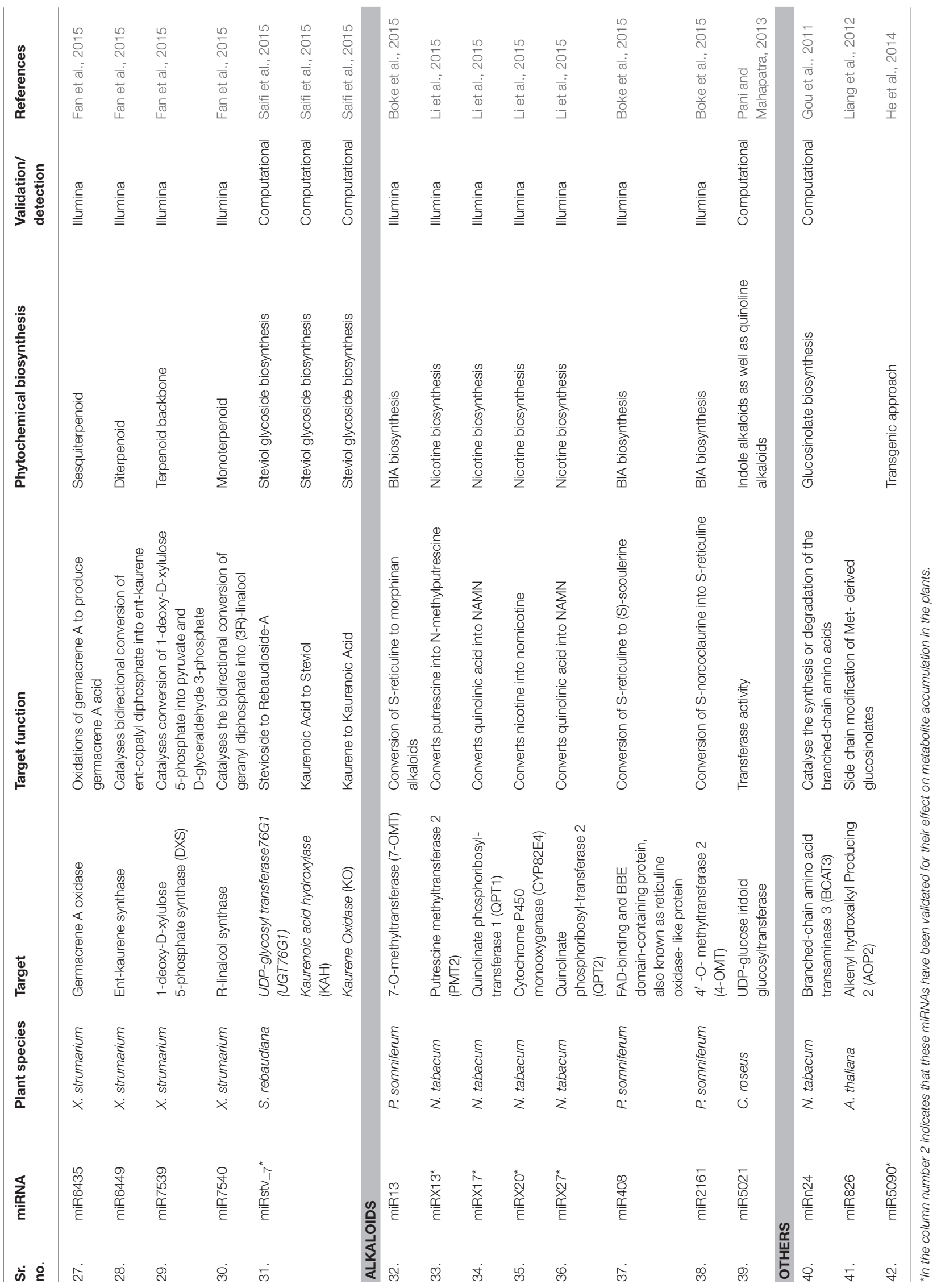
oxidase-like protein which converts S-reticuline to (S)-scoulerine in the BIA pathway. Endogenous target mimicry (eTM) of miRNAs disturbs the function of corresponding miRNAs by inhibiting binding of miRNAs with their authentic target genes (Franco-Zorrilla et al., 2007). Therefore, Li and his co-workers in 2015 have demonstrated that nta-eTMX27 inhibits the expression and function of nta-miRX27 which targets mRNA of quinolinate phosphoribosyl transferase 2 (QPT2) genes leading to enhanced nicotine biosynthesis in the topping treated tobacco. The most recent report by Mao et al. (2017) shows the regulatory role of miR156 targeting SPL9 in the biosynthesis of glucosinolates, which are secondary metabolites functioning as defense metabolites against insect herbivores and pathogens. The SPL9 interacts with JA ZIM-domain (JAZ) proteins, including JAZ3 to control jasmonate synthesis. Increased level of jasmonate further promotes the biosynthesis of glucosinolates. In addition, several other workers have reported numerous miRNAs along with their target genes involved in the alkaloid biosynthetic pathway in P. hexandrum (Biswas et al., 2016), R. serpentina (Prakash et al., 2016), and C. roseus (Pani and Mahapatra, 2013) using computational approaches.

\section{MODULATING SECONDARY METABOLITES VS. PRIMARY METABOLITE BIOSYNTHETIC PATHWAYS THROUGH miRNAs}

Unlike secondary metabolites, primary metabolites are required by plants at every stage of their growth and development. And also, the precursor molecules for secondary metabolite biosynthesis are channelized from primary metabolites. Regulation of primary metabolite biosynthetic pathways is well explored at transcriptional, post-transcriptional and now at DNA level, but secondary metabolic pathway are limited at the transcriptional level and recently at post-transcriptional level (miRNA). Therefore, till now, most of the work has focussed on the role of miRNAs during primary metabolism of growth and development. Recently, these miRNAs of primary metabolism along with some other miRNAs are being reported for their crucial role during secondary metabolism, for example, the SPL-miRNA156 system (Gou et al., 2011). Similarly, miR-4995 targets 3-deoxy-7-phosphoheptulonate synthase gene involved in the first step of phenylpropanoid pathway for picrosides I biosynthesis (Vashisht et al., 2015). Being the first enzyme of the pathway, this enzyme holds the key to the progress of pathway as its down-regulation can affect the production of cinnamic acid, thereby affecting picrosides I content. Taking into account the regulatory roles of miRNAs, modification in the expression of such miRNAs would be a promising approach to modulate the biosynthesis of secondary metabolites in plants. SPL9 and TCP3 transcription factors play a major role in secondary metabolism regulation (Gou et al., 2011; Li and Zachgo, 2013) and therefore miRNAs targeting these genes would be an ideal candidate for such approach (Bulgakov and Avramenko, 2015). Nevertheless, identifying and understanding the spatial and temporal expression schema of other miRNAs that might regulate the flux movement at the branch point of primary vs. secondary metabolic pathway and/or secondary metabolic pathway alone would help designing better strategies to favor the biosynthesis of economically important secondary metabolites.

\section{CONCLUSION AND FUTURE DIRECTIONS}

Owing to the diversity of the biosynthetic pathway of the secondary metabolites and their biological significance in both plants and human, exploring the regulatory schema of the pathway is crucial. Despite the role of miRNAs during different biotic and abiotic stresses and plant developmental processes, their role in regulating the biosynthesis of secondary metabolites had just started accumulating and it further requires intense and focussed work. Studies on identification of miRNAs and their targets at all possible steps of the pathway and characterizing significant miRNAs-target pairs using reverse genetics is one prime area to decipher the functions of miRNAs. Deep sequencing technologies and the modern computational approaches for miRNA predictions has resulted in the accumulation of huge data on miRNAs. Despite the availability of many computational algorithms, miRNA target identification is still a major challenge. Many miRNA targets which have miRNA binding sites with seed mismatches could not be identified due to the inability of computational tools (Doran and Strauss, 2007). Presently, most of the miRNA target predictions, consider mRNA $3^{\prime}$ UTRs and therefore the genes that are regulated by miRNA through binding in the region other than 3' UTRs could not be identified (Place et al., 2008; Tay et al., 2008). The miRNAs are part of complex regulatory networks where a single miRNA control lots of genes. Thus, modulation of single miRNA expression could result in complicated biological consequences (Lee et al., 2014). This complexity makes functional validation by knock-out or overexpression of these predicted miRNAs a challenging issue.

Furthermore, understanding the DNA methylation profiles of plant genomes and their interaction with miRNAs and selfregulation of miRNAs would be an interesting future area of research. In addition, Work on the potential of herbal medicinederived miRNAs in regulating human health or targeting genes associated with diseases are another emerging area. Such studies would help metabolic engineering of the entire biosynthetic pathway for generating novel phytochemicals or for producing desired combinations of such secondary metabolites.

\section{AUTHOR CONTRIBUTIONS}

$\mathrm{OG}$ and $\mathrm{AD}$ conceived the idea and designed the manuscript, OG, SB, SK, NM collected literature and wrote the manuscript, OG, SK, and AD critically evaluated the manuscript. All authors approved the manuscript.

\section{ACKNOWLEDGMENTS}

The authors apologize to colleagues whose work could not be cited due to space constraints. 


\section{REFERENCES}

Biswas, S., Hazra, S., and Chattopadhyay, S. (2016). Identification of conserved miRNAs and their putative target genes in Podophyllum hexandrum (Himalayan Mayapple). Plant Gene 6, 82-89. doi: 10.1016/j.plgene.2016. 04.002

Boke, H., Ozhuner, E., Turktas, M., Parmaksiz, I., Ozcan, S., and Unver, T. (2015). Regulation of the alkaloid biosynthesis by miRNA in opium poppy. Plant Biotechnol. J. 13, 409-420. doi: 10.1111/pbi.12346

Buer, C. S., Imin, N., and Djordjevic, M. A. (2010). Flavonoids: new roles for old molecules. J. Integr. Plant Biol. 52, 98-111. doi: 10.1111/j.1744-7909.2010.00905.x

Bulgakov, V. P., and Avramenko, T. V. (2015). New opportunities for the regulation of secondary metabolism in plants: focus on microRNAs. Biotechnol. Lett. 37, 1719-1727. doi: 10.1007/s10529-015-1863-8

Cho, Y. B., Jones, S. I., and Vodkin, L. (2013). The transition from primary siRNAs to amplified secondary siRNAs that regulate chalcone synthase during development of Glycine max seed coats. PLoS ONE 8:e76954. doi: 10.1371/journal.pone.0076954

Cui, L. G., Shan, J. X., Shi, M., Gao, J. P., and Lin, H. X. (2014). The miR156SPL9-DFR pathway coordinates the relationship between development and abiotic stress tolerance in plants. Plant J. 80, 1108-1117. doi: 10.1111/tpj. 12712

Doran, J., and Strauss, W. M. (2007). Bio-informatic trends for the determination of miRNA-target interactions in mammals. DNA Cell Biol. 26, 353-360. doi: 10.1089/dna.2006.0546

Dudareva, N., Negre, F., Nagegowda, D. A., and Orlova, I. (2006). Plant volatiles: recent advances and future perspectives. Crit. Rev. Plant Sci. 25, 417-440. doi: $10.1080 / 07352680600899973$

Fan, R., Li, Y., Li, C., and Zhang, Y. (2015). Differential microRNA analysis of glandular trichomes and young leaves in Xanthium strumarium L. reveals their putative roles in regulating terpenoid biosynthesis. PLOS ONE 10:e0139002. doi: 10.1371/journal.pone.0139002

Fraenkel, G. S. (1959). The raison d'être of secondary plant substances these odd chemicals arose as a means of protecting plants from insects and now guide insects to food. Science 129, 1466-1470. doi: 10.1126/science.129.3361.1466

Franco-Zorrilla, J. M., Valli, A., Todesco, M., Mateos, I., Puga, M. I., RubioSomoza, I., et al. (2007). Target mimicry provides a new mechanism for regulation of microRNA activity. Nat. Genet. 39, 1033-1037. doi: $10.1038 / \mathrm{ng} 2079$

Gou, J. Y., Felippes, F. F., Liu, C. J., Weigel, D., and Wang, J. W. (2011). Negative regulation of anthocyanin biosynthesis in Arabidopsis by a miR156-targeted SPL transcription factor. Plant Cell 23, 1512-1522. doi: 10.1105/tpc.111.084525

Gupta, O. P., Sharma, P., Gupta, R. K., and Sharma, I. (2014a). MicroRNA mediated regulation of metal toxicity in plants: present status and future perspectives. Plant Mol. Biol. 84, 1-18. doi: 10.1007/s11103-013-0120-6

Gupta, O. P., Sharma, P., Gupta, R. K., and Sharma, I. (2014b). Current status on role of miRNAs during plant-fungus interaction. Physiol. Mol. Plant Pathol. 85, 1-7. doi: 10.1016/j.pmpp.2013.10.002

He, H., Liang, G., Li, Y., Wang, F., and Yu, D. (2014). Two young microRNAs originating from target duplication mediate nitrogen starvation adaptation via regulation of glucosinolate synthesis in Arabidopsis thaliana. Plant Physiol. 164, 853-865. doi: 10.1104/pp.113.228635

Jones-Rhoades, M. W., Bartel, D. P., and Bartel, B. (2006). MicroRNAs and their regulatory roles in plants. Annu. Rev. Plant Biol. 57, 19-53. doi: 10.1146/annurev.arplant.57.032905.105218

Kirtikar, K. R., and Basu, B. D. (1918). Indian Medicinal Plants. Bahadurganj: Sudhindra Nath Basu.

Lee, H. M., Nguyen, D. T., and Lu, L. F. (2014). Progress and challenge of microRNA research in immunity. Front. Genet. 12:178. doi: $10.3389 /$ fgene.2014.00178

Lepiniec, L., Debeaujon, I., Routaboul, J. M., Baudry, A., Pourcel, L., Nesi, N., et al. (2006). Genetics and biochemistry of seed flavonoids. Annu. Rev. Plant Biol. 57, 405-430. doi: 10.1146/annurev.arplant.57.032905.105252

Li, F., Wang, W., Zhao, N., Xiao, B., Cao, P., Wu, X., et al. (2015). Regulation of nicotine biosynthesis by endogenous target mimicry of microRNA in tobacco. Plant Physiol. 169, 1062-1071. doi: 10.1104/pp.15.00649
Li, S. (2014). Transcriptional control of flavonoid biosynthesis: fine-tuning of the MYB-bHLH-WD40 (MBW) complex. Plant Signal. Behav. 9:e27522. doi: $10.4161 / \mathrm{psb} .27522$

Li, S., and Zachgo, S. (2013). TCP3 interacts with R2R3-MYB proteins, promotes flavonoid biosynthesis and negatively regulates the auxin response in Arabidopsis thaliana. Plant J. 76, 901-913. doi: 10.1111/tpj.12348

Liang, G., He, H., and Yu, D. (2012). Identification of nitrogen starvationresponsive microRNAs in Arabidopsis thaliana. PLoS ONE 7:e48951. doi: 10.1371/journal.pone.0048951

Luo, Y., Zhang, X., Luo, Z., Zhang, Q., and Liu, J. (2015). Identification and characterization of microRNAs from Chinese pollination constant nonastringent persimmon using high-throughput sequencing. BMC Plant Biol. 15:11 doi: 10.1186/s12870-014-0400-6

Mahajan, V., Mahajan, A., Pagoch, S. S., Bedi, Y. S., and Gandhi, S. G. (2011). microRNA mediated regulation of plant secondary metabolism: an In silico analysis. J. Nat. Sci. Biol. Med. 2, 44-45

Mao, Y. B., Liu, Y. Q., Chen, D. Y., Chen, F. Y., Fang, X., Hong, G. J., et al. (2017). Jasmonate response decay and defense metabolite accumulation contributes to age-regulated dynamics of plant insect resistance. Nat. Commun. 9:13925. doi: $10.1038 /$ ncomms13925

Pani, A., and Mahapatra, R. N. (2013). Computational identification of microRNAs and their targets in Catharanthus roseus expressed sequence tags. Genomics Data 1, 2-6. doi: 10.1016/j.gdata.2013.06.001

Place, R. F., Li, L. C., Pookot, D., Noonan, E. J., and Dahiya, R. (2008). MicroRNA373 induces expression of genes with complementary promoter sequences. Proc. Natl. Acad. Sci. U.S.A. 105, 1608-1613. doi: 10.1073/pnas.07075 94105

Prakash, P., Rajakani, R., and Gupta, V. (2016). Transcriptome-wide identification of Rauvolfia serpentina microRNAs and prediction of their potential targets. Comput. Biol. Chem. 61, 62-74. doi: 10.1016/j.compbiolchem.2015. 12.002

Quattrocchio, F., Verweij, W., Kroon, A., Spelt, C., Mol, J., and Koes, R. (2006). PH4 of Petunia is an R2R3 MYB protein that activates vacuolar acidification through interactions with basic-helix-loop-helix transcription factors of the anthocyanin pathway. Plant Cell 18, 1274-1291. doi: 10.1105/tpc.105.034041

Riese, M., Hohmann, S., Saedler, H., Munster, T., and Huijser, P. (2007). Comparative analysis of the SBP-box gene families in $P$. patens and seed plants. Gene 401, 28-37. doi: 10.1016/j.gene.2007.06.018

Saif, M., Nasrullah, N., Ahmad, M. M., Ali, A., Khan, J. A., and Abdin, M. Z. (2015). In silico analysis and expression profiling of miRNAs targeting genes of steviol glycosides biosynthetic pathway and their relationship with steviol glycosides content in different tissues of Stevia rebaudiana. Plant Physiol. Biochem. 94, 57-64. doi: 10.1016/j.plaphy.2015.05.009

Samuni-Blank, M., Izhaki, I., Dearing, M. D., Gerchman, Y., Trabelcy, B., Lotan, A., et al. (2012). Intraspecific directed deterrence by the mustard oil bomb in a desert plant. Curr. Biol. 22, 1218-1220. doi: 10.1016/j.cub.2012.04.051

Santelia, D., Henrichs, S., Vincenzetti, V., Sauer, M., Bigler, L., Klein, M., et al. (2008). Flavonoids redirect PIN-mediated polar auxin fluxes during root gravitropic responses. J. Biol. Chem. 283, 31218-31226. doi: $10.1074 / \mathrm{jbc} . \mathrm{m} 710122200$

Sharma, D., Tiwari, M., Pandey, A., Bhatia, C., Sharma, A., and Trivedi, P. K. (2016). MicroRNA858 is a potential regulator of phenylpropanoid pathway and plant development Plant Physiol. 171, 944-959. doi: 10.1104/pp.15.01831

Shriram, V., Kumar, V., Devarumath, R. M., Khare, T. S., and Wani, S. H. (2016). miRNAs as potential targets for abiotic stress tolerance in plants. Front. Plant Sci. 7:817. doi: 10.3389/fpls.2016.00817

Singh, N., Srivastava, S., and Sharma, A. (2016b). Identification and analysis of miRNAs and their targets in ginger using bioinformatics approach. Gene 575 , 570-576. doi: 10.1016/j.gene.2015.09.036

Singh, N., Srivastava, S., Shasany, A. K., and Sharma, A. (2016a). Identification of miRNAs and their targets involved in the secondary metabolic pathways of Mentha spp. Comput. Biol. Chem. 64, 154-162. doi: 10.1016/j.compbiolchem.2016.06.004

Stamp, N. (2003). Out of the quagmire of plant defense hypotheses. Q. Rev. Biol. 78, 23-55. doi: 10.1086/367580

Stracke, R., Ishihara, H., Huep, G., Barsch, A., Mehrtens, F., Niehaus, K., et al. (2007). Differential regulation of closely related R2R3-MYB transcription 
factors controls flavonol accumulation in different parts of the Arabidopsis thaliana seedling. Plant J. 50, 660-666. doi: 10.1111/j.1365-313X.2007.03078.x

Tang, W., and Eisenbrand, G. (1992). Chinese Drugs of Plant Origin, Chemistry, Pharmacology and Use in Traditional and Modern Medicine. Berlin: Springer Verlag. 1065.

Tay, Y., Zhang, J., Thomson, A. M., Lim, B., and Rigoutsos, I. (2008). MicroRNAs to Nanog, Oct4 and Sox2 coding regions modulate embryonic stem cell differentiation. Nature 455, 1124-1128. doi: 10.1038/nature07299

Taylor, L. P., and Grotewold, E. (2005). Flavonoids as developmental regulators. Curr. Opin. Plant Biol. 8, 317-323. doi: 10.1016/j.pbi.2005.03.005

Tuteja, J. H., Zabala, G., Varala, K., Hudson, M., and Vodkin, L. O. (2009). Endogenous, tissue-specific short interfering RNAs silence the chalcone synthase gene family in Glycine max seed coats. Plant Cell 21, 3063-3077. doi: $10.1105 /$ tpc. 109.069856

Vashisht, I., Mishra, P., Pal, T., Chanumolu, S., Singh, T. R., and Chauhan, R. S. (2015). Mining NGS transcriptomes for miRNAs and dissecting their role in regulating growth, development, and secondary metabolites production in different organs of a medicinal herb, Picrorhiza kurroa. Planta 241, 1255-1268. doi: 10.1007/s00425-015-2255-y

Verpoorte, R. (1998). Exploration of nature's chemodiversity: the role of secondary metabolites as leads in drug development. Drug Discov. Today 3, 232-238. doi: 10.1016/S1359-6446(97)01167-7

Wagner, K. H., and Elmadfa, I. (2003). Biological relevance of terpenoids. Overview focusing on mono-di- and tetraterpenes. Ann. Nutr. Metab. 47, 95-106. doi: 10.1159/000070030

Wu, G., Park, M. Y., Conway, S. R., Wang, J. W., Weigel, D., and Poethig, R. S. (2009). The sequential action of miR156 and miR172 regulates developmental timing in Arabidopsis. Cell 138, 750-759. doi: 10.1016/j.cell.2009.06.031

Wu, J., Wang, D., Liu, Y., Wang, L., Qiao, X., and Zhang, S. (2014). Identification of miRNAs involved in pear fruit development and quality. BMC Genomics 3:953. doi: 10.1186/1471-2164-15-953

Xing, S., Salinas, M., Hohmann, S., Berndtgen, R., and Huijser, P. (2010). miR156-targeted and nontargeted SBP-box transcription factors act in concert to secure male fertility in Arabidopsis. Plant Cell 22, 3935-3950. doi: $10.1105 /$ tpc. 110.079343

Xu, X., Jiang, Q., Ma, X., Ying, Q., Shen, B., Qian, Y., et al. (2014). Deep sequencing identifies tissue-specific microRNAs and their target genes involving in the biosynthesis of tanshinones in Salvia miltiorrhiza. PLoS ONE 9:e111679. doi: 10.1371/journal.pone.0111679

Yamaguchi, A., Wu, M. F., Yang, L., Wu, G., Poethig, R. S., and Wagner, D. (2009). The microRNA-regulated SBP-Box transcription factor SPL3 is a direct upstream activator of LEAFY, FRUITFULL, and APETALA1. Dev. Cell 17, 268-278. doi: 10.1016/j.devcel.2009.06.007

Yang, R., Zeng, Y., Yi, X., Zhao, L., and Zhang, Y. (2015). Small RNA deep sequencing reveals the important role of microRNAs in the halophyte Halostachys caspica. Plant Biotechnol. J. 13, 395-408. doi: 10.1111/pbi. 12337

Yu, N., Cai, W. J., Wang, S., Shan, C. M., Wang, L. J., and Chen, X. Y. (2010). Temporal control of trichome distribution by microRNA156-targeted SPL genes in Arabidopsis thaliana. Plant Cell 22, 2322-2335. doi: 10.1105/tpc.109.072579

Yu, Z. X., Wang, L. J., Zhao, B., Shan, C. M., Zhang, Y. H., Chen, D. F., et al. (2015). Progressive regulation of sesquiterpene biosynthesis in Arabidopsis and Patchouli (Pogostemon cablin) by the miR156-targeted SPL transcription factors. Mol. Plant 8, 98-110. doi: 10.1016/j.molp.2014.11.002

Zhang, M., Dong, Y., Nie, L., Lu, M., Fu, C., and Yu, L. (2015). High-throughput sequencing reveals miRNA effects on the primary and secondary production properties in long-term subcultured Taxus cells. Front. Plant Sci. 6:604. doi: 10.3389/fpls.2015.00604

Ziegler, J., and Facchini, P. J. (2008). Alkaloid biosynthesis: metabolism and trafficking. Annu. Rev. Plant Biol. 59, 735-769. doi: 10.1146/annurev.arplant.59.032607.092730

Conflict of Interest Statement: The authors declare that the research was conducted in the absence of any commercial or financial relationships that could be construed as a potential conflict of interest.

Copyright (c) 2017 Gupta, Karkute, Banerjee, Meena and Dahuja. This is an openaccess article distributed under the terms of the Creative Commons Attribution License (CC BY). The use, distribution or reproduction in other forums is permitted, provided the original author(s) or licensor are credited and that the original publication in this journal is cited, in accordance with accepted academic practice. No use, distribution or reproduction is permitted which does not comply with these terms. 